\title{
Neurasthenia in a longitudinal cohort study of young adults
}

\author{
K. MERIKANGAS ${ }^{1}$ AND J. ANGST \\ From the Genetic Epidemiology Research Unit, Yale University School of Medicine, New Haven, CT, USA; \\ and Psychiatric University Hospital, Zurich, Switzerland
}

SYNOPSIS This study examines the concept of neurasthenia in a longitudinal cohort of young adults selected from a community sample of the canton of Zurich, Switzerland. The major focus is on the validity of the case definition of neurasthenia. Close approximations of the proposed descriptive and research definitions of the ICD-10 are employed as well as the concept of "irritable weakness' as described in 1831 by Kraus (1926-1932).

The prevalence of neurasthenia defined according to the ICD- 10 criteria was: $1 \%$ across 10 years and $0.9 \%$ in 1988 for a duration criterion of $\geqslant 3$ months; and $8.1 \%$ across 10 years and $12 \%$ in 1988 for a duration criterion of $\geqslant 1$ month. The duration criterion of $\geqslant 3$ months appeared to be excessively restrictive to represent individuals with neurasthenia in the community. Subjects with 1 month episodes of neurasthenia exhibited sufficient differences from controls and similarities to subjects with anxiety or depressive disorders to justify a 1 month duration criterion for neurasthenia in community samples.

The clinical significance of neurasthenia was indicated by the magnitude of subjective distress, and occupational and social impairment reported by the majority of the cases. Prospective assessment of the longitudinal course of neurasthenia revealed that approximately $50 \%$ of the cases continued to exhibit this disorder at follow-up. Our findings suggest that neurasthenia is equally likely to represent an early manifestation of affective illness as it is a consequence in those neurasthenic subjects who exhibited comorbid affective disorders. The magnitude, chronicity, impairment, longitudinal stability and distinction from anxiety and depression associated with this condition in the general population, suggest that neurasthenia is an important diagnostic entity for which additional validation studies should be undertaken.

\section{INTRODUCTION}

With the recent focus on chronic fatigue syndrome, there has been a resurgence of interest in the concept of neurasthenia (Costa e Silva \& De Girolamo, 1990; Wessely, 1991). Although Beard, the American neurologist, is credited with the introduction of the term 'neurasthenia' in 1869 to describe a syndrome characterized by exhaustion of the nervous system, various forms of nervous asthenia have been described throughout the history of medicine (Costa e Silva \& De Girolamo, 1990).

\footnotetext{
1 Address for correspondence: Dr K. Merikangas, Genetic Epidemiology Research Unit, Yale University School of Medicine, 40 Temple Street, New Haven, CT 06510, USA.
}

\section{Definition}

Beard's description of this syndrome included the following criteria: general malaise, debility of all the functions, poor appetite, abiding weakness in the back and spine, fugitive neuralgic pains, hysteria, insomnia, hypochondria, disinclination for consecutive mental labour, severe and weakening attacks of sick headache, and other analogous symptoms. He also required that anaemia and other organic diseases be excluded (Beard, 1869).

The definition of neurasthenia has generally been vague and broad. Although the key features of physical and mental fatigue are generally included in most definitions, the specific concomitant symptoms are highly variable. The 
Table 1. International Classification of Disease10 criteria for F48.0 neurasthenia

A Either of the following must be present:

(1) Persistent and distressing complaints of feelings of exhaustion after minor mental effort (such as performing or attempting to perform everyday tasks that do not require unusual mental effort);

(2) Persistent and distressing complaints of feelings of fatigue and bodily weakness after minor physical effort.

B At least one of the following symptoms must be present:

(1) feelings of muscular aches and pains

(2) dizziness

(3) tension headaches

(4) sleep disturbance

(5) inability to relax

(6) irritability.

$C$ The patient is unable to recover from the symptoms in criterion A (l) or (2) by means of rest, relaxation, or entertainment.

$D$ The duration of the disorder is at least 3 months.

E The disorder does not occur in the presence of organic emotionally labile disorder, post-encephalitic syndrome, postconcussional syndrome, mood (affective) disorders, panic disorder, or generalized anxiety disorder.

major difference between some of the more recent definitions of the condition and the descriptions of the neurologists of the nineteenth and early twentieth centuries is the inclusion of 'nervous irritability' as a key feature of the condition. A cluster analysis of the symptoms of subjects from the general community conducted by Angst \& Koch (1991) yielded the same three distinct factors that have been the key features of clinical descriptions of neurasthenia, namely, physical exhaustion, mental fatigue and nervous irritability.

Although the symptoms of chronic fatigue syndrome (CFS) are quite similar to those cited above for neurasthenia, the major difference between the two definitions is the requirement of myalgia for CFS (Wessely, 1991). The aetiology of CFS has often been attributed to viral infections as in the post-viral fatigue syndromes (i.e. chronic Epstein-Barr infection, Tobi et al. 1982); however, evidence that the characteristic physical and mental fatigue are induced by viral infection is still lacking (Holmes et al. 1988).

Aetiological theories of neurasthenia are nearly as numerous as the physicians who described the disease: horseback riding (Hippocrates, in Roccatagliata, 1973); metabolic factors including glucose metabolism, pituitary dysfunction, and thyroid abnormalities
(Mirouze, 1962); life stress (Beard, 1869); drug abuse (Beard, 1869); sexual excesses (Freud, 1895); genetic factors (Möbius, 1894; Costa e Silva \& De Girolamo, 1990); personality factors; and psychiatric disorders, particularly depression. However, most contemporary formulations require exclusion of organic abnormalities in order to diagnose neurasthenia.

\section{Association between neurasthenia and somatic disturbances}

A variety of non-psychiatric syndromes have been associated with neurasthenia. Beard (1869) described stomach disturbances, migraine, menstrual disorders, back pain, and respiratory problems. Although most of the diagnostic criteria for neurasthenia rule out discrete organic causes of the syndrome, the above-cited somatic disturbances tend to comprise symptoms rather than pathognomonic manifestations of a specific disease.

\section{Association between neurasthenia and psychiatric disorders}

An association between neurasthenia and the 'neurotic' psychiatric disorders has been reported in both descriptive literature and systematic studies of clinical samples. The three most frequently cited disorders are depression, anxiety, and somatization. In the classic essay in which he first described 'Anxiety Neurosis', Freud contended that Beard's formulation of the concept of neurasthenia was too broad to be clinically meaningful. His definition of genuine neurasthenia excluded the hereditary nervous disorders and the 'pseudo-neurasthenias' induced by physical diseases, as well as melancholia and anxiety neurosis (Beard, 1869). Thus, Freud provided the first clear discrimination between neurasthenia and anxiety neurosis and melancholia (Freud, 1895). Freud's observation was recently confirmed by a multivariate analysis by Goldberg et al. (1987), who demonstrated that a symptom cluster characterized by fatigue can be distinguished from those with anxiety and depression.

The proportions of neurasthenics who also exhibit a psychiatric disorder range from onehalf to three-quarters in psychiatric samples (Allan, 1944; Taerk et al. 1987; Manu et al. 1988; Strauss, 1988; Swartz, 1988; Kruesi et al. 1989; Wessely \& Powell, 1989; Hickie et al. 
1990), and from one-third to one-half in primary care settings (Katon \& Walker, 1993). Katon \& Walker (1993) reported that the association between fatigue and anxiety and depression increases as one moves from the community to primary care to tertiary care facilities. This suggests that neurasthenics with psychiatric disorders are more likely to seek treatment than those without comorbid conditions.

Previous analyses of this sample revealed that $50 \%$ of the neurasthenic subjects had a history of anxiety or depression. Moreover, the persons with extended neurasthenia had nearly twice the degree of comorbidity with the latter disorders than did those with recurrent brief neurasthenia (Angst \& Koch, 1991).

\section{Personality and neurasthenia}

In a study of the pre-morbid personality of neurasthenics who had sought treatment for this condition, Nystrom \& Lindegard (1975) found that although sub-validity, or psychasthenia, was associated with neurasthenia, even greater associations emerged between psychasthenia and anxiety and depression. This suggests that personality factors comprise non-specific and independent risk factors for the subsequent development of these syndromes. More recently, Blakely et al. (1991) examined psychiatric symptoms and personality in patients with CFS and chronic pain compared to normal controls. In classifying the patients on both the symptom and personality scales, they found several clusters of CFS patients, one of which had no evidence of psychiatric or personality disorder. Moreover, their data suggested that emotionality is a predisposing factor rather than a reaction to neurasthenia (Blakely et al. 1991). However, these findings require replication in a prospective study of a more systematic sample.

\section{Treatment of neurasthenia}

In accordance with the multiple causative factors which have been implicated for neurasthenia, numerous forms of treatment have been suggested as well. For example, Beard (1869) recommended general electrization (i.e. electric shocks applied over the head and spine), whereas Vittoz (1903) applied 'brain control'. Antidepressants are the most widely employed agents in the contemporary treatment of neurasthenia or CFS (Wessely, 1991).

\section{Current studies of neurasthenia}

The diagnostic criteria for research on neurasthenia in the most recent version of the International Classification of Disease, Edition 10 (WHO, 1993) are presented in Table 1. The chief criteria are as follows: $(a)$ persistent fatigue after ordinary tasks; $(b)$ one of six symptoms; $(c)$ inability to recover after rest; $(d)$ duration of at least 3 months; and $(e)$ exclusion of concomitant anxiety and depressive syndromes, and specific organic syndromes. The distinction between neurasthenia and anxiety/depression described by Freud (1895) has been adopted in the ICD-10 as well; concurrent anxiety and depression preclude a diagnosis of neurasthenia.

The emergence of CFS as a significant disease entity has led to the generation of numerous studies investigating the clinical phenomenology and associated features of this condition. Community studies of fatigue and fatigability have provided preliminary evidence of the magnitude of this complaint in the general population. Although variation in sampling methods precludes derivation of accurate aggregate rates across studies, the current prevalence of fatigue is approximately $24 \%$ in community studies and $15 \%$ in primary care settings (Lewis \& Wessely, 1992). Although there is a greater proportion of women with fatigue, there is still a substantial proportion of males who complain of fatigue. The most important finding from these studies is the substantial degree of global dysfunction and chronicity associated with fatigue (Kroenke et al. 1988; David et al. 1990; Cathébras et al. 1992). However, the degree to which the findings regarding the symptom of fatigue generalize with those with the syndrome of neurasthenia needs to be examined.

Although there are several studies currently investigating the prevalence of the ICD-10 disorders in the community and in primary care settings under the auspices of the WHO, to our knowledge, there are no published data from epidemiological samples on the magnitude or correlates of this condition in the general population. The major purpose of the present study is to assess the validity of the case definition of neurasthenia. Close approximations of the proposed descriptive and research definitions of the ICD-10 (WHO, 1993) are employed as well as the concept of 'irritable weakness' as de- 
scribed by Kraus (1926-1932). Therefore, the goals of the present study were as follows.

(1) To estimate the magnitude and demographic distribution of neurasthenia in a prospective study of a cohort selected from the general population.

(2) To test the applicability of the ICD-10 definition of neurasthenia in an epidemiological sample through systematic assessment of the inclusion and exclusion criteria and their validity.

(3) To examine the association and longitudinal course of psychiatric disorders and neurasthenia.

(4) To examine the clinical and social manifestations of neurasthenia and its longitudinal stability.

\section{METHOD}

\section{Subjects}

The subjects of this report are a cohort of 29-30year-olds from Zurich, Switzerland. The sample was originally selected in 1978 according to scores on a screening test of current symptoms, the Symptom Checklist 90 (SCL-90-R), from which high and low risk groups were selected. The SCL-90-R was developed by Derogatis (1977) as a self-report inventory about current psychiatric symptoms. It is a 90 -item multidimensional symptom inventory that yields scores on nine symptom dimensions. The sample was first interviewed in 1979 at age 20, with three subsequent interviews in 1981, 1986, and 1988. The subjects in this report are comprised of 424 subjects ( 200 males and 224 females) who were interviewed directly in 1988. A more detailed description of the sampling procedures is given by Angst $e t$ al. (1984) and Merikangas et al. (1990).

\section{Procedure}

A direct interview, the Structured Psychopathological Interview and Rating of the Social Consequences for Epidemiology (SPIKE), was administered by psychiatric residents and clinical psychologists with extensive clinical training. This interview schedule assesses a number of somatic syndromes, including headache, gastrointestinal, cardiovascular, and respiratory syndromes, as well as psychological syndromes including depression, phobia, obsessive-com- pulsive, anxiety, and substance abuse (Angst $e t$ al. 1984). Psychiatric diagnoses were made according to both the DSM-III and the DSMIII-R Criteria (American Psychiatric Association, 1980, 1987). Screening probes were administered for each section and symptoms, duration, frequency, severity, and treatment history and impairment are assessed for every positive answer. Personal and family history of the syndromes were also assessed for all subjects, irrespective of endorsement of the diagnostic screening question for each section.

\section{Symptoms of neurasthenia}

Symptoms of neurasthenia were collected at all four interviews. Because the interviews were developed prior to the introduction of the ICD10 , only two of the symptomatic criteria in Section B were collected in the diagnostic interview, namely sleep disturbances and irritability. Therefore, we required at least one of these symptoms for the diagnosis of neurasthenia. The other symptoms of neurasthenia were assessed in the SCL-90, which was administered at the time of each of the four interviews. However, the vast majority of subjects complaining of persistent weakness and fatigue met more than half of the symptomatic criteria for neurasthenia. Organic causes of neurasthenic symptoms could not be excluded in our data.

Impairment and severity were assessed in two ways: ( 1 ) as an analogue rating from $0-100$, with 0 representing no impairment (i.e. no interference with daily activities or the absence of suffering or distress from the symptoms), and a score of 100 representing complete incapacitation or extreme suffering or distress from the symptoms; and (2) as a categorical scale from $1-5$, with descriptions to anchor each level of severity or impairment.

\section{Assessment of personality}

The Freiburg Personality Inventory (FPI) is a 212-item personality questionnaire designed to measure 9 primary factors including nervousness, aggressivity, depressiveness, excitability, sociability, temperament and striving for dominance, inhibition and frankness (Fahrenberg $e t$ al. 1970). In addition to three secondary factor provided by the test developers, three factors derived from a factor analysis of very large 
Table 2. Weighted longitudinal and 1-year prevalence rates of neurasthenia by components of ICD-10 definition

\begin{tabular}{|c|c|c|c|c|c|}
\hline Neurasthenia groups & 1979 & 1981 & 1986 & 1988 & Total \\
\hline Symptoms $*$ Duration $>3$ months ${ }^{*}+$ Anxiety $/$ depression $\dagger$ & 0.7 & 0.1 & $1 \cdot 1$ & $2 \cdot 1$ & $3 \cdot 7$ \\
\hline Symptoms + Duration $>3$ months $\ddagger$ & $1 \cdot 0$ & $0 \cdot 6$ & $0 \cdot 2$ & $0 \cdot 9$ & $1 \cdot 0$ \\
\hline Symptoms + Anxiety/depression & $2 \cdot 3$ & 1.5 & 5.9 & $2 \cdot 5$ & $11 \cdot 4$ \\
\hline Symptoms only* & $2 \cdot 6$ & 1.8 & $5 \cdot 4$ & $7 \cdot 6$ & 7.0 \\
\hline Anxiety/depression, No symptoms* & $11 \cdot 8$ & $17 \cdot 1$ & $17 \cdot 3$ & $16 \cdot 6$ & $25 \cdot 3$ \\
\hline None of the above & 81.6 & 78.9 & $70 \cdot 1$ & $70 \cdot 3$ & 51.6 \\
\hline
\end{tabular}

* One of ICD-10 symptom criteria for neurasthenia.

$\dagger$ DSM-III-R anxiety or affective disorder.

$\ddagger$ ICD-10 definition of neurasthenia.

samples at the Zurich site were also employed, namely extraversion, autonomic lability, and aggressivity (Angst \& Clayton, 1986).

\section{Statistical analyses}

In the present study, the statistical analyses that were conducted to test the significance of the differences for comparisons of the categorical variables were $\chi^{2}$ corrected for continuity. Analyses of variance were applied to the continuous variables using the general linear models procedure of the Statistical Analysis System (Reinhardt \& Winston, 1985). Group mean differences were compared with the Duncan Multiple Range Test, which corrects for chance (Duncan, 1975).

\section{RESULTS}

\section{Assessment of diagnostic criteria}

Table 2 presents the weighted and longitudinal rates of neurasthenia according to the step-wise application of the ICD-10 criteria for neurasthenia. The figures in the second row labelled 'symptoms and duration $\geqslant 3$ months' represent the subjects who met all of the ICD-10 criteria for neurasthenia (i.e. persistent fatigue, $\geqslant 1$ of the 6 symptoms, inability to recover, plus $\geqslant 3$ months duration and the absence of concomitant anxiety or depressive disorders) at each of the 4 interviews and across the entire study interval. Inspection of longitudinal trends shown in Table 2 revealed that the rates of neurasthenia were quite stable over 10 years at $1 \%$. However, there was an increase over time in the proportion of subjects who reported symptoms of neurasthenia as the sample aged from 20 to 30 . Inspection of the sex ratio across the 10 years of follow-up revealed that the prevalence of neurasthenia was equal among males and females during the initial stages of the study, whereas females exhibited 1.6-fold greater rates of neurasthenia than males during the later interviews.

Application of successive criteria of exclusion of subjects with anxiety or depressive disorders and duration of $\geqslant 3$ months to the proportion of subjects with neurasthenic symptoms yielded a dramatic decrease in the longitudinal rates of neurasthenia from $23 \cdot 1 \%$ to $1 \%$. This indicates that nearly $75 \%$ of individuals complaining of persistent and distressing fatigue and weakness with symptomatic manifestations are excluded from the diagnosis of neurasthenia. Because the 3 month duration criterion yielded groups that were too small for meaningful analysis, a 1 month duration was employed in subsequent analyses. The major focus of this paper is therefore on determining whether differences exist between pure and comorbid cases of neurasthenia. There were no differences between the four groups in demographic characteristics including marital status, education, number of children and social class.

\section{Symptoms}

The non-criterial symptoms of neurasthenia collected in the diagnostic interview and selfreported criterial symptoms according to comorbid anxiety and depression in neurasthenic subject, at the most recent interview are presented in Fig. 1. No major differences in symptom frequencies emerged between the comorbid and non-comorbid neurasthenics. The large proportion of neurasthenic subjects who 


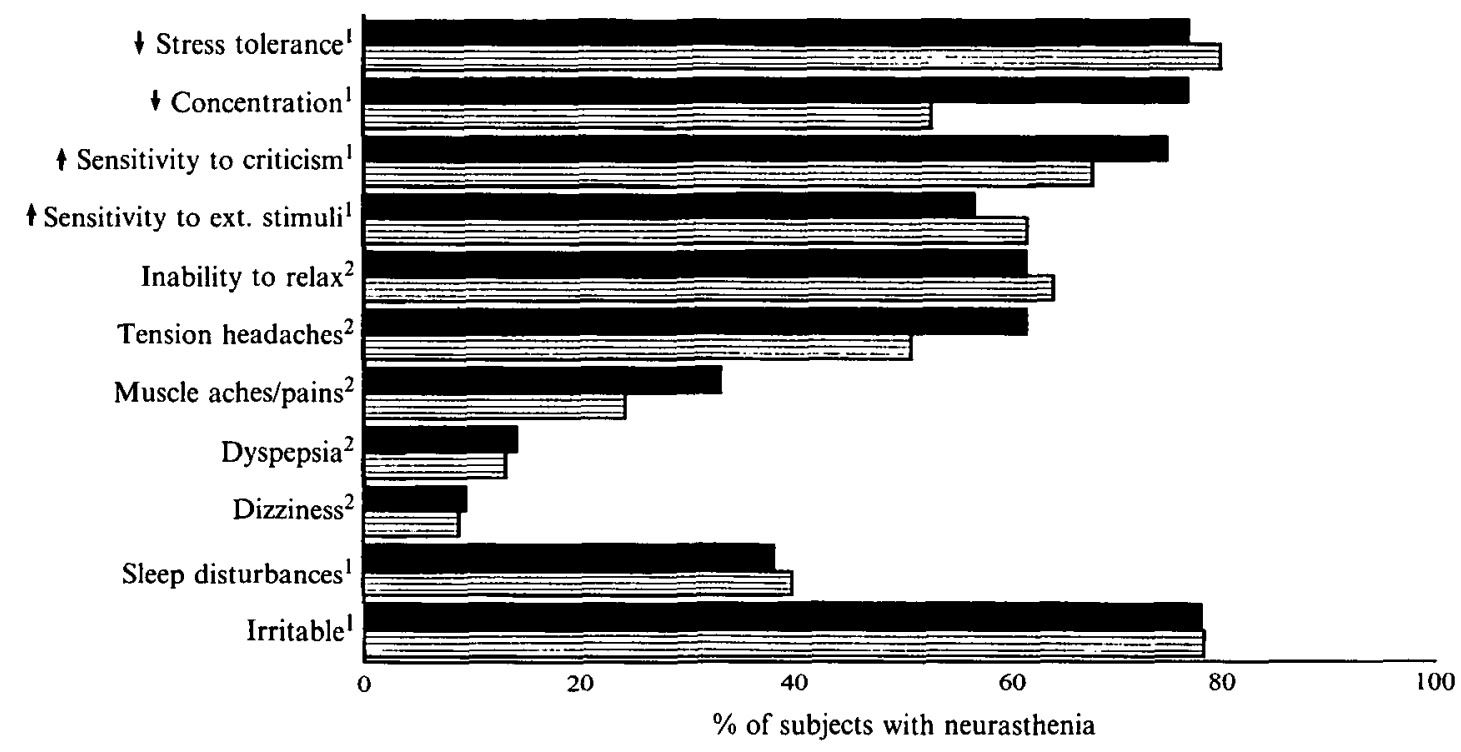

FIG. 1. Symptoms of neurasthenia by neurasthenia comorbidity groups in 1988. (ם, Anxiety/depression + neurasthenia; 目, neurasthenia only; ${ }^{1}=$ interview; and ${ }^{2}=$ SCL-90 self-report.)

Table 3. SCL-90 scores $\dagger$ in neurasthenia by comorbidity groups in 1988

\begin{tabular}{|c|c|c|c|c|}
\hline . & $\begin{array}{c}\text { Neurasthenia } \\
+ \text { Anx/dep } \\
(N=32) \\
\text { Mean (s.D.) }\end{array}$ & $\begin{array}{c}\text { Neurasthenia } \\
\text { only } \\
(N=51) \\
\text { Mean (S.D.) }\end{array}$ & $\begin{array}{c}\text { Anx/dep } \\
\text { only } \\
(N=74) \\
\text { Mean (s.D.) }\end{array}$ & $\begin{array}{c}\text { Neither } \\
(N=267) \\
\text { Mean (s.D.) }\end{array}$ \\
\hline \multicolumn{5}{|l|}{ Scale scores } \\
\hline Anxiety & $60 \cdot 3(11 \cdot 43)$ & $56 \cdot 1(8 \cdot 3)$ & $55.9(14.13)$ & $50.4(11 \cdot 28)^{* *}$ \\
\hline Depression & $66.1(11.73)$ & $60 \cdot 7(7 \cdot 2)$ & $61 \cdot 1(12 \cdot 0)$ & $53.8(10.92)^{* *}$ \\
\hline Hostility & $55.9(13.96)$ & $55.6(10.0)$ & $56.3(10-7)$ & $51.5(10-33) * *$ \\
\hline Interpersonal sensitivity & $66.4(13.40)$ & $60 \cdot 8(9 \cdot 14)$ & $60.8(13.66)$ & $54.9(11.56)^{* *}$ \\
\hline Obsessive compulsive & $63 \cdot 3(13.40)$ & $54.9(10.8)$ & $56.4(12.99)$ & $50.9(11 \cdot 56)^{* *}$ \\
\hline Paranoia & $62.6(10.66)$ & $57 \cdot 1(10 \cdot 13)$ & $56.7(11.62)$ & $53.2(11.55)^{* *}$ \\
\hline Phobia & $60.9(11.43)$ & $55 \cdot 1(12 \cdot 4)$ & $55 \cdot 8(12.26)$ & $50.3(11.37)^{* * *}$ \\
\hline Psychoticism & $64 \cdot 2(11 \cdot 96)$ & $57.7(10.04)$ & $57.0(12.02)$ & $53.1(10.41)^{* *}$ \\
\hline \multicolumn{5}{|l|}{ Factor scores } \\
\hline Somatic instability & $65 \cdot 2(9 \cdot 68)$ & $53 \cdot 2(10 \cdot 98)$ & $52.8(10.67)$ & $48.6(9.42)^{* *}$ \\
\hline Emotional instability & $60 \cdot 9(10 \cdot 14)$ & $55.6(6.99)$ & $56.3(9.91)$ & $50.8(9.43)^{* *}$ \\
\hline Vegetative instability & $58.4(7.91)$ & $56 \cdot 1(8 \cdot 26)$ & $55.8(8.79)$ & $51 \cdot 2(8.11)^{* *}$ \\
\hline Global severity index & $65 \cdot 0(11 \cdot 30)$ & $58.9(7.39)$ & $60 \cdot 2(11 \cdot 31)$ & $53.8(9.17)^{* *}$ \\
\hline
\end{tabular}

$\dagger$, transformed $($ mean $=50$, S.D. $=10)$.

** $P<0.01$.

reported decreased stress tolerance, and increased sensitivity to criticism and to external stimuli (i.e. $82 \%, 73 \%$ and $67 \%$, respectively) suggests that these phenomena may constitute additional features of neurasthenia. In contrast, the criteria of muscle aches and pains, dyspepsia, and dizziness were reported by less than one-third of the subjects with neurasthenia, suggesting that they may not comprise valid clinical features of this condition.
The factor and scale scores of the SCL-90 in the neurasthenia groups and controls are shown in Table 3. All three groups of affected subjects differed significantly from the unaffected subjects on all of the scale scores and factor scores of the SCL-90. The comorbid group exhibited the greatest scores on all scales, whereas the groups of subjects with either neurasthenia alone or anxiety/depression alone had nearly identical scores on all scales. This suggests a lack of 
Table 4. Clinical features of neurasthenia by comorbidity in 1988

\begin{tabular}{|c|c|c|c|}
\hline & \multirow{2}{*}{$\begin{array}{c}\begin{array}{c}\text { Neurasthenia } \\
\text { + Anx/dep }\end{array} \\
(N=32)\end{array}$} & \multicolumn{2}{|c|}{$\begin{array}{l}\text { Neurasthenia } \\
\text { only }\end{array}$} \\
\hline & & \multicolumn{2}{|c|}{$(N=51)$} \\
\hline \multicolumn{4}{|c|}{ Maximal duration/episode (past year \%) } \\
\hline$\leqslant 1$ week & $28 \cdot 1$ & $66 \cdot 7$ & \\
\hline$>1$ week-1 month & $\begin{array}{r}6 \cdot 3 \\
65 \cdot 6\end{array}$ & 19.6 & ** \\
\hline \multicolumn{4}{|l|}{ Subjective distress } \\
\hline mean (S.D.) & $\begin{array}{c}25 \cdot 8 \\
(25 \cdot 7)\end{array}$ & $\begin{array}{c}53 \cdot 7 \\
(28 \cdot 0)\end{array}$ & \\
\hline \multicolumn{4}{|l|}{ Attribution (\%) } \\
\hline Physical & $22 \cdot 6$ & $30 \cdot 2$ & \\
\hline Psychological & $96 \cdot 8$ & $72 \cdot 1$ & $* *$ \\
\hline \multicolumn{4}{|l|}{ Impairment (\%) } \\
\hline Occupational & $80 \cdot 6$ & $79 \cdot 1$ & \\
\hline Social & 93.5 & 86.0 & \\
\hline Dimensional rating, mean (S.D.) & $\begin{array}{c}39.7 \\
(29 \cdot 0)\end{array}$ & $\begin{array}{c}33 \cdot 9 \\
(25 \cdot 6)\end{array}$ & \\
\hline \multicolumn{4}{|l|}{ Professional treatment } \\
\hline Past year $(\%)$ & 41.9 & $23 \cdot 3$ & \\
\hline Lifetime, mean (S.D.) & $\begin{array}{c}0.7 \\
(0.83)\end{array}$ & $\begin{array}{c}0.3 \\
(0.69)\end{array}$ & * \\
\hline
\end{tabular}

specificity of the SCL-90 scale scores for both neurasthenia and affective disorders.

\section{Clinical features}

The clinical features of neurasthenia in the two groups of subjects during the year prior to the most recent interview are presented in Table 4. Patterns of duration of neurasthenic episodes differed for the comorbid and non-comorbid groups. The majority of subjects in the noncomorbid group reported episodes of approximately one week, but occurring frequently over the one year period. In contrast, the majority of the comorbid subjects reported longer episodes, lasting an average of 1-2 months, but with less frequency than the non-comorbid group.

The degree of subjective distress attributed to neurasthenia was similar for the two groups of subjects. The magnitude of the distress was rated on an analogue scale from 0 , indicating no distress, to 100 for extreme distress. The high mean levels found in both groups suggest that the symptoms of neurasthenia are associated with a severe degree of distress in all of the subjects who meet the criteria for this syndrome.

Likewise, there was a remarkable degree of subjective and occupational impairment associated with neurasthenia among all affected persons. Approximately $80 \%$ of the cases reported that neurasthenia symptoms interfered with their ability to pursue their usual occupational activities. The mean level of 80 (range $0-100)$ suggests that the complications of neurasthenia were sufficient to inhibit routine daily activities. Similar findings emerged for the dimensional measures of impairment in both occupation and social activity.

The proportion of subjects who had sought treatment differed significantly between the comorbid and non-comorbid groups. The treatment rates among the comorbid cases were similar to those of the anxiety and depression group, with $42 \%$ reporting having sought

Table 5. Rates of somatic and psychiatric disorders by neurasthenia comorbidity groups in 1988

\begin{tabular}{|c|c|c|c|c|}
\hline & $\begin{array}{l}\text { Neurasthenia } \\
+ \text { Anx } / \text { dep } \\
(N=32)\end{array}$ & $\begin{array}{c}\text { Neurasthenia } \\
\text { only } \\
(N=57)\end{array}$ & $\begin{array}{c}\text { Anx/dep } \\
\text { only } \\
(N=74)\end{array}$ & $\begin{array}{l}\text { Neither } \\
(N=267)\end{array}$ \\
\hline \multicolumn{5}{|l|}{ Somatic disturbances (\%) } \\
\hline Back & $50 \cdot 0$ & $37 \cdot 3$ & 37.8 & $31 \cdot 8$ \\
\hline Circulation & 21.9 & $23 \cdot 5$ & 13.5 & $9 \cdot 4^{* *}$ \\
\hline Menstrual (females only) & $52 \cdot 9$ & $35 \cdot 1$ & $44 \cdot 4$ & $34 \cdot 3$ \\
\hline Migraine & 32.0 & 43.8 & $25 \cdot 8$ & $23 \cdot 3^{*}$ \\
\hline Respiratory/heart & $28 \cdot 1$ & 15.7 & $10 \cdot 8$ & $6 \cdot 7^{* *}$ \\
\hline Stomach & $43 \cdot 8$ & $25 \cdot 5$ & $20 \cdot 3$ & $10 \cdot 5^{* *}$ \\
\hline \multicolumn{5}{|l|}{ Psychiatric disorders $(\%)$} \\
\hline Alcoholism & $9 \cdot 4$ & 3.9 & $8 \cdot 1$ & $2 \cdot 3^{*}$ \\
\hline Cannabis abuse & $3 \cdot 1$ & $2 \cdot 0$ & $2 \cdot 8$ & $0 \cdot 4$ \\
\hline Tobacco dependence & $62 \cdot 6$ & $41 \cdot 2$ & $41 \cdot 7$ & $25 \cdot 8^{* *}$ \\
\hline Agoraphobia & $6 \cdot 3$ & $2 \cdot 0$ & $2 \cdot 8$ & 1.5 \\
\hline Simple phobia & $6 \cdot 3$ & 11.8 & 10.8 & 8.6 \\
\hline Social phobia & $9 \cdot 4$ & 39 & $4 \cdot 2$ & $2 \cdot 7$ \\
\hline
\end{tabular}

\footnotetext{
${ }^{*} P<0.05 ;{ }^{* *} P<0.01$.
} 
Table 6. FPI scores by neurasthenia comorbidity groups in 1988

\begin{tabular}{|c|c|c|c|c|}
\hline & $\begin{array}{c}\text { Neurasthenia } \\
+ \text { Anx/dep } \\
(N=32) \\
\text { Mean (S.D.) }\end{array}$ & $\begin{array}{c}\text { Neurasthenia } \\
\text { only } \\
(N=51) \\
\text { Mean (s.D.) }\end{array}$ & $\begin{array}{c}\text { Anx/dep } \\
\text { only } \\
(N=74) \\
\text { Mean (s.D.) }\end{array}$ & $\begin{array}{c}\text { Neither } \\
(N=267) \\
\text { Mean (s.D.) }\end{array}$ \\
\hline \multicolumn{5}{|l|}{ Scale scores } \\
\hline Nervousness & $22 \cdot 9(8 \cdot 77)$ & $20 \cdot 5(7.63)$ & $18 \cdot 5(7.25)$ & $15 \cdot 1(5.52)^{* *}$ \\
\hline Aggressiveness & $21.5(7.51)$ & $16.4(5.92)$ & $18 \cdot 3(6.95)$ & $15.2(6.82)^{* *}$ \\
\hline Depression & $22.0(8.30)$ & $16 \cdot 6(6 \cdot 98)$ & $17.4(8.04)$ & $12 \cdot 1(6 \cdot 33)^{* *}$ \\
\hline Irritability & $25.9(8.07)$ & $23.0(8.04)$ & $21.9(8 \cdot 18)$ & $19.5(8.00)^{* *}$ \\
\hline Sociability & $20.3(7.63)$ & $18 \cdot 6(8 \cdot 16)$ & $19.5(8.03)$ & $20.6(7.28)$ \\
\hline Resilience & $13.4(9.41)$ & $13.7(7.76)$ & $12.8(8.82)$ & $16.6(7.57)^{* *}$ \\
\hline Aggressive reaction & $19.1(7.63)$ & $17 \cdot 1(6 \cdot 97)$ & $17 \cdot 3(7 \cdot 16)$ & $15.4(7.43)^{* *}$ \\
\hline Inhibition & $21.9(9.01)$ & $21.7(8.89)$ & $21.4(8.67)$ & $18.2(8.40)^{* *}$ \\
\hline Openness & $19 \cdot 7(6 \cdot 14)$ & $19.0(7.55)$ & $18 \cdot 0(8 \cdot 01)$ & $16 \cdot 7(8.06)$ \\
\hline \multicolumn{5}{|l|}{ Factor scales } \\
\hline Aggressiveness & $22.2(7 \cdot 72)$ & $18.9(6.74)$ & $18.8(7.07)$ & $16.6(7.20)^{* *}$ \\
\hline Extraversion & $17 \cdot 3(8.08)$ & $16 \cdot 3(8.00)$ & $17.1(7.96)$ & $19.2(7.59) *$ \\
\hline Neuroticism (autonomic lability) & $23.4(7.65)$ & $20 \cdot 1(7.44)$ & $18.5(7.31)$ & $14.7(5.92)^{* * *}$ \\
\hline
\end{tabular}

$\dagger t$ transformed (mean $=20$, s.D. $=8$ ).
$* P<0.05 ;{ }^{* *} P<0.01 ;{ }^{* *} P<0.001$.

Neurasthenia only $(N=33)$

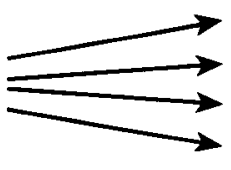

$14 \%$ Neurasthenia + Affective/anxiety disorder

$31 \%$ Neurasthenia only

$21 \%$ Affective/anxiety disorder only

$34 \%$ Neither

Affective or anxiety disorder only $(N=110)$

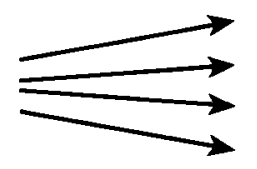

$8 \%$ Neurasthenia + Affective/anxiety disorder

$25 \%$ Neurasthenia only

$17 \%$ Affective/anxiety disorder only

$50 \%$ Neither

FIG. 2. Ten-year follow-up of subjects with neurasthenia only or affective/anxiety disorder only.

professional treatment for their condition. In contrast, only $23 \%$ of the pure neurasthenics had sought professional treatment.

\section{Comorbidity}

Table 5 presents the cross-sectional association between neurasthenia and other somatic and psychiatric syndromes at age 30 . Because the concept of neurasthenia includes both physical and mental weakness, the SPIKE interview, which assessed a variety of somatic conditions as well as psychological conditions, was an ideal source of data on this condition. Neurasthenia was associated with numerous somatic conditions including migraine, stomach disturbances, circulation problems, cardiac and respiratory disturbances. However, other conditions assessed, including back problems, were not associated with neurasthenia, thereby suggesting that these subjects are not simply exhibiting a tendency to somaticize across all systems.

The associations between neurasthenia and the major psychiatric disorders are also shown in Table 5. Because major depression, dysthymia, panic disorder, and generalized anxiety were included in the group definitions, the analyses 
shown in this table include only the phobic disorders and substance abuse. Alcoholism was elevated only among the subjects with depression/anxiety disorders with or without neurasthenia, with a four-fold elevation in the rates of alcoholism compared to that of controls. For all other disorders except simple phobia, the non-comorbid groups exhibited intermediate rates between the comorbid and the controls.

\section{Personality}

The factor and scale scores on the FPI at the most recent interview according to the neurasthenic comorbidity groups are shown in Table 6. The neurasthenics had elevated scores on nearly all scales with the exception of the depression score. The results of the factor score analyses revealed that the neurasthenics were characterized by somatic symptoms, autonomic instability, and irritability. The comorbid group exhibited higher scores on all of these dimensions than the pure neurasthenics. These findings validate the symptoms endorsed by the subjects during the diagnostic interview, because they were assessed completely independently of the subjects report of the cluster of symptoms which characterized neurasthenia.

\section{Course}

The course of neurasthenia among subjects without comorbidity at the initial interview in 1979 is presented in Fig. 2. Neurasthenia was much more stable over the longitudinal course than the affective disorders. Nearly one-half of the persons with neurasthenia at the time of the initial interview continued to exhibit neurasthenia at the follow-up, whereas only $25 \%$ of the subjects with affective disorders reported affective disorders at the follow-up. In contrast, subjects with pure neurasthenia were equally likely to develop an affective or anxiety disorder during the follow-up (i.e. $35 \%$ ) as were those with only an affective or anxiety disorder at the initial interview (i.e. $33 \%$ ).

Another indicator of the course of neurasthenia assessed in the interview was the number of years over the entire study that the subjects reported symptoms of neurasthenia. The average duration of neurasthenia across the entire 10-year-period was about 4 years, with the comorbid group reporting longer duration (mean $=4.5$ years) than the non-comorbid group (mean $=3.5$ years). The age of onset of neurasthenia was also assessed retrospectively at the most recent interview. The average age of onset was estimated to be significantly earlier among the comorbid than in the pure neurasthenics, with the comorbid subjects reporting an average age of onset of 17.9 and the non-comorbid subjects reporting an average of $19 \cdot 7$.

\section{DISCUSSION}

The prevalence of neurasthenia defined according to the ICD-10 criteria was $1 \%$ across 10 years and $0.9 \%$ in 1988 for a duration of $\geqslant 3$ months, and $8.1 \%$ across 10 years and $12 \%$ in 1988 for a duration of $\geqslant 1$ month. The duration criterion of $\geqslant 3$ months appeared to be excessively restrictive to represent individuals with neurasthenia in the community. Subjects with 1 month episodes of neurasthenia exhibited sufficient differences from controls and subjects with anxiety or depressive disorders alone to justify a 1 month duration criterion for neurasthenia in community samples. However, differences between neurasthenia alone and those with comorbid anxiety or depressive disorders supported the exclusion of these conditions from the criteria for neurasthenia. Although there was an excess of females over the longitudinal course, cross-sectional data indicate that a substantial proportion of males also suffer from this condition.

Despite the low prevalence of neurasthenic episodes of greater than 3 months duration, all of the subjects reported chronicity of the condition. That is, the average case with $>1$ month duration reported the presence of neurasthenic symptoms during an average of 4 years of the 10 years of the study. This is quite remarkable in light of the relatively youthful age of this cohort.

The results of the present study also suggest that the validity of the definition would be enhanced by the inclusion of the dimension of sensitivity to stress. The interview data from the present study confirmed the results of an earlier cluster analysis (Angst \& Koch, 1991) which suggested that neurasthenia should be broadened to include the concept of stress intolerance, which characterized the majority of persons with neurasthenia.

The stability of the sub-typing according to 
comorbidity for anxiety and depression was confirmed by prospective data on the longitudinal course over a decade. The pure neurasthenics were discriminated from the comorbid group by differences in treatment rates, patterns of comorbidity, and the longitudinal stability of neurasthenia. In contrast to the majority of previous studies of clinical samples which consider neurasthenia as a manifestation of underlying depression and anxiety syndromes (e.g. Wilson et al. 1983), these findings suggest that there is no characteristic pattern of the order of onset of neurasthenia and affective and anxiety disorders. In the present study, the onset of neurasthenia was equally likely to pre-date as it was to post-date the onset of these comorbid conditions. In contrast to most previous clinical studies, Hickie et al. (1990) reported that the onset of psychological disturbances tended to post-date the onset of fatigue in patients with CFS.

The severity of neurasthenia was indicated by the magnitude of subjective distress, and both occupational and social impairment which was reported by $80 \%$ of the cases. Likewise, in a study of the treated prevalence of CFS, Lloyd $e t$ al. (1990) reported that $43 \%$ of the patients with this syndrome were completely incapacitated. Kroenke et al. (1988) also noted the striking degree of dysfunction associated with fatigue in their primary care sample. Indeed, the impact of fatigue was similar to that of survivors of myocardial infection, sudden cardiac death and hypothyroidism. Aside from the magnitude of the suffering experienced by persons with this condition, the medical expenses and decreased occupational productivity are associated with an enormous indirect cost as well.

The chronicity of neurasthenia was demonstrated by the continued manifestation of disorders in more than half of the subjects over a decade, at an average duration of 4 to 5 years. Similar findings emerged from 1 year prospective follow-ups of primary care patients with complaints of fatigue. Kroenke et al. (1988) found that fatigue persisted in $72 \%$ of their patients after 1 year and Cathébras et al. (1992) estimated that $50-66 \%$ of their sample exhibited chronicity over 1 year. The magnitude, chronicity and impairment associated with this condition in the general population, as well as the substantial proportion of persons in whom manifestation of this syndrome is distinct from anxiety of depressive disorders, suggest that neurasthenia is a valid diagnostic entity, and should be considered for inclusion in American diagnostic systems.

Neurasthenia is frequently encountered in diverse medical specialities including neurology, psychiatry, internal medicine, cardiology, gastroenterology, and rheumatology, with no speciality claiming neurasthenia within its sole domain. This is not surprising because the disorder itself has moved between specialities since it was first described. For example, Beard (1869) was a neurologist, Griesinger (1845) an internist, Freud (1895) a psychiatrist and neurologist and Kielholz (1957) a psychiatrist. Although heterogeneous aetiology is likely given the magnitude and protean manifestations of this condition, the treatment of choice is currently antidepressant medications, after detectable somatic aetiologies have been excluded by appropriate clinical or laboratory tests (Wessely \& Powell, 1989).

Despite severe levels of impairment and distress, however, few of the pure neurasthenics had actually consulted a professional of any speciality for this disorder. This explanation is supported by the finding that the neurasthenics with affective disorders in the present study had sought treatment more often, either because of the severity of the concomitant manifestations of symptoms in both domains, or the relative availability of treatment and public awareness of depression. The small proportion of pure neurasthenics who seek treatment may be associated with the mixture of somatic and psychological features of this syndrome. In contrast to subjects from treated samples (see Wessely \& Powell, 1989), the majority of the neurasthenics in the present study attributed their symptoms to psychological and somatic factors. The psychological manifestations, when taken together with a lack of disturbance in a specific organ system, may impede identification of an appropriate source of treatment. Resistance to psychiatric treatment may also be an important component of the infrequency with which these individuals seek professional help.

These findings underscore the need for empirical data on this syndrome from both epidemiological and treatment settings. Epidemiological data on a wider age range of subjects 
are critical to investigate the magnitude, impact, and comorbidity in order to identify sources of heterogeneity in its expression, as well as for planning for health services. Further work is necessary to study the case definition across diverse settings. There is also a critical need for systematic treatment studies and prospective follow-up studies of clinical samples. The current follow-up of this sample at age 35 will yield information on the stability of neurasthenia and differential patterns of course among persons with underlying psychiatric disorders.

This work was supported in part by Research Scientist Development Award MH00499 from the United States Public Health Service to Dr Merikangas; and Grant 32-33580.92 from the Swiss National Science Foundation (Professor Angst).

\section{REFERENCES}

Allan, F. (1944). The differential diagnosis of weakness and fatigue. New England Journal of Medicine 231, 414-418.

American Psychiatric Association Committee on Nomenclature and Statistics (1980). Diagnostic and Statistical Manual of Mental Disorders, 3rd edn. American Psychiatric Association: Washington, DC.

American Psychiatric Association Committee on Nomenclature and Statistics (1987). Diagnostic and Statistical Mamual of Mental Disorders, 3rd edn, Revised. American Psychiatric Association: Washington, DC.

Angst J. \& Clayton, P. J. (1986). Premorbid personality of depressive, bipolar, and schizophrenic patients with special reference to suicidal issues. Comprehensive Psychiatry 27, 511-532.

Angst, J., Dobler-Mikola, A. \& Binder, J. (1984). The Zurich study - a prospective epidemiological study of depressive, neurotic and psychosomatic syndromes: I. Problem, methodology European Archives of Psychiatry and Neurological Sciences 234, 13-20.

Angst, J. \& Koch, R. (1991). Neurasthenia in young adults. In Problems of Psychiatry in General Practice: Neurasthenia, Obsessive-Compulsive Disorder. Advances in Treatment of Depression. Teaching and Training of the GP (ed. M. Gastpar and P. Kielholz), pp. 37-48. Hogrefe \& Huber: Toronto.

Beard. G. (1869). Neurasthenia, or nervous exhaustion. Boston Medical and Surgical Journal III, 217-221.

Blakely, A. A.. Howard, R. C., Sosich, R. M., Murdoch, J. C. Menkes, D. B. \& Spears, G. F. S. (1991). Psychiatry symptoms, personality and ways of coping in chronic fatigue syndrome. Psychological Medicine 21, 347-362.

Cathẻbras, P. J., Robbins, J. M.. Kirmayer, L. J. \& Hayton, B. C. (1992). Fatigue in primary care: prevalence, psychiatric comorbidity, illness behavior and outcome. Journal of General Internal Medicine 7, 276-286.

Costa e Silva, J. A. \& De Girolamo, G. (1990). Neurasthenia: history of a concept. In Psychological Disorders in General Medical Settings (ed. N. Sartorius, D. Goldberg, G. de Girolamo, J. Costa e Silva, Y. Lecrubier and U. Wittchen), pp. 69-8I. Hogrefe \& Huber: Toronto.

David. A.. Pelosi, A., McDonald, E.. Stephens, D., Ledger, D., Rathbone, R. \& Mann, A. (1990). Tired, weak, or in need of rest: fatigue among general practice attenders. British Medical Journal 301. 1199-1202.
Derogatis, R. L. (1977). Symptom Checklist 90, R-Version Manual I: Scoring. Administration and Procedures for the SCL-90. Johns Hopkins University Press: Baltimore.

Duncan, D. B. (1975). $t$-Tests and intervals for comparisons suggested by the data. Biometrics 31, 339-359.

Fahrenberg, J., Selg, H. \& Hampel, R. (1970). Das Freiburger Persontichkeitcinventar. Hogrete: Göttingen.

Freud, S. (1895) On the Grounds for Detaching a Particular Syndrome from Neurasthenia under the Description 'Anxiety Neurosis', 3rd edn. Hogarth Press: London.

Goldberg, D., Bridges, K., Duncan-Jones, P. \& Grayson, D. (1987). Dimensions of neuroses seen in primary care settings. Psychological Medicine 17, 461-470.

Griesinger, W. (1945). Die Pathologie und Therapie der psychischen Krankheiten und Zustandsbilder. Hans Huber: Vienna.

Hickie, I., Lloyd, A., Wakefield, D. \& Parker, G. (1990). The psychiatric status of patients with the chronic fatigue syndrome. British Journal of Psychiatry 156, 534-540.

Holmes, G. P., Kaplan, J. E., Gantz, N. M., Komaroff, A. L., Dobois, R., Cunningham-Rundles, C., Pahwa, S., Tosato, G., Zegans, L. S., Purtilo, D. T., Brown, N., Schooley, R. T. \& Brus, I. (1988). Chronic fatigue syndrome: a working definition. Amals of Internal Medicine 108, 387-389.

Katon, W. J. \& Walker, E. A. (1993). The relationship of chronic fatigue to psychiatric illness in community, primary care, and tertiary care samples. In Chronic Fatigue Syndrome, pp. 193-211. (Ciba Foundation Symposium 173.) Wiley: Chichester.

Kielholz, P. (1957). Diagnostik und Therapie der depressiven Zustandsbilder. Schweizerische Medizinische Wochenschrifi 87, 107.

Kraus, L. A. (1926-32). Kridisch-Etymologisches Medicinisches Lexikon, 2nd edn. R. Deuerlich: Göttingen.

Kroenke, K., Wood, D. R., Mangeisdorff, A. D., Meier, N. J. \& Powell, J. B. (1988). Chronic fatigue in primary care: prevalence, patient characteristics, and outcome. Journal of the American Medical Association 260, 929-934.

Kruesi, M. J. P., Dale, J. \& Straus, S. E. (1989). Psychiatric diagnosis in patients who have chronic fatigue syndrome. Journal of Clinical Psychiatry 50, 53-56.

Lewis, G. \& Wessely, S. (1992). The epidemiology of fatigue: more questions than answers. Journal of Epidemiology and Community Health 46, 92-97.

Lloyd, A., Hickie, I., Boughton, C. R., Spence, O. \& Wakefield, D. (1990). Prevalence of chronic fatigue syndrome in an Australian population. Medical Journal of Australia 153, 522-528.

Manu, P., Matthews, D. A. \& Lane, T. J. (1988). The mental health of patients with a chief complaint of chronic fatigue: a prospective evaluation and follow-up. Archives of Internal Medicine 148, 2213-2217.

Merikangas, K. R., Angst, J. \& Isler, H., (1990). Migraine and psychopathology: results of the Zurich cohort study of young adults. Archives of General Psychiatry 47, 849-853.

Mirouze, J. (1962). Problèmes psychosomatiques et hormonaux de la fatigue. Assisses de Médecine 3, 1-4.

Möbius, P. (1894). Neurologische Beitrage. Abel: Leipzig.

Nystrom, S. \& Lindegard, B. (1975). Predisposition for mental syndromes: a study comparing predisposition for depression, neurasthenia and anxiety state. Acta Psychiatrica Scandinavica 51. 69-76.

Reinhardt, P. \& Winston, X. (1985). SAS Supplemental Library User's Guide. SAS Institute: Cary, NC.

Roccatagliata, G. (1973). Storia della Psichiatria Antica. Hocpli: Milan.

Straus, S. E. (1988). The chronic mononucleosis syndrome. Journal of Infectious Diseases 57, 405-412.

Swartz, M. N. (1988). The chronic fatigue syndrome- one entity of many (editorial)? New England Journal of Medicine 319, 1726-1728.

Taerk, G. S., Toner, B. B., Salit, I. S., Garfinkel, P. E. \& Ozersky, S. (1987). Depression in patients with neuromyasthenia (benign myalgic encephalomyelitis). Imernational Journal of Psychiatric Medicine 17, 49-56. 
Tobi, M., Morag, A., Ravid, Z. Chowers, 1., Feldman-Weiss, V., Michaeli, Y., Ben-Chetrit, E., Shalit, M. \& Knobler, H. (1982) Prolonged atypical illness associated with serologic evidence of Epstein-Barr virus infection. Lancet i, 61-64.

Vittoz, R. (1913). Treatment of Neurasthenia by Means of Brain Control, 2nd edn. Longmans, Green and Co: London.

Wessely, S. (1991). History of postviral fatigue syndrome. British Medical Bulletin 47, 919-941.

Wessely, S. \& Powell, R. (1989). Fatigue syndromes: a comparison of chronic 'post-viral' fatigue with neuromuscular and affective disorders. Journal of Neurology, Neurosurgery and Psychiatry 42, 940-948.

Wilson, D. R., Widmer, R. B., Cadoret, R. J. \& Judiesch, K. (1983). Somatic symptoms: a major feature of depression in a family practice. Journal of Affective Disorders 5, 199-207.

World Health Organization (1993). The ICD-10 Classification of Mental and Behavioural Disorders: Diagnostic Criteria for Research. World Health Organization: Geneva, 